\title{
Study of heavy-ion induced fission for heavy-element synthesis
}

\author{
K. Nishio ${ }^{1, a}$, H. Ikezoe ${ }^{1}$, S. Hofmann"2,3, F.P. Heßberger ${ }^{2}$, D. Ackermann², S. Antalic ${ }^{4}$, \\ Y. Aritomo ${ }^{1,5}$, V.F. Comas ${ }^{2,6}$, Ch.E. Düllman², A. Gorshkov ${ }^{7}$, R. Graeger ${ }^{7}$, S. Heinz ${ }^{2}$, \\ J.A. Heredia ${ }^{2,6}$, K. Hirose ${ }^{1,8}$, J. Khuyagbaatar ${ }^{2}$, B. Kindler ${ }^{2}$, I. Kojouharov², B. Lommel ${ }^{2}$, H. Makii ${ }^{1}$, \\ R. Mann ${ }^{2}$, S. Mitsuoka ${ }^{1}$, Y. Nagame ${ }^{1}$, I. Nishinaka ${ }^{1}$, T. Ohtsuki ${ }^{8}$, A.G. Popeko ${ }^{5}$, S. Saro ${ }^{4}$, \\ M. Schädel ${ }^{2}$, A. Türler ${ }^{7}$, Y. Wakabayashi ${ }^{1,9}$, Y. Watanabe ${ }^{10}$, A. Yakushev ${ }^{7}$, and A.V. Yeremin ${ }^{5}$ \\ ${ }^{1}$ Japan Atomic Energy Agency, Tokai, Ibaraki, Japan \\ ${ }^{2}$ GSI Helmholzzentrum für Schwerionenforschung, Darmstadt, Germany \\ ${ }^{3}$ Institute für Physik, Goethe-Universität Frankfurt, Germany \\ ${ }^{4}$ Comenius University, Bratislava, Slovakia \\ ${ }^{5}$ Flerov Laboratory of Nuclear Reactions, Dubna, Russia \\ ${ }^{6}$ Higher Institute of Technologies and Applied Sciences, Habana, Cuba \\ ${ }^{7}$ Institute für Radiochemie, Technische Universität München, Garching, Germany \\ ${ }^{8}$ Laboratory of Nuclear Science, Tohoku University, Sendai, Japan \\ ${ }^{9}$ Nishina-center, RIKEN, Wako, Saitama, Japan \\ ${ }^{10}$ High Energy Accelerator Research Organization (KEK), Tsukuba, Japan
}

\begin{abstract}
Fission fragment mass distributions were measured in heavy-ion induced fissions using ${ }^{238} \mathrm{U}$ target nucleus. The measured mass distributions changed drastically with incident energy. The results are explained by a change of the ratio between fusion and qasifission with nuclear orientation. A calculation based on a fluctuation dissipation model reproduced the mass distributions and their incident energy dependence. Fusion probability was determined in the analysis, and the values were consistent with those determined from the evaporation residue cross sections.
\end{abstract}

\section{Introduction}

Experiments to produce superheavy nuclei (SHN) have been carried out by using heavy-ion fusion and evaporation reactions [1-3]. Prediction of the cross sections for SHN is important to make an experimental plan and explore this region of chart of nuclei. The reaction proceeds in three steps; (1) penetration of the Coulomb barrier between two colliding nuclei (capture), (2) formation of a compound nucleus after nuclear contact (3) survival of the excited compound nucleus by particle evaporation against fission. The first step, penetrating the Coulomb barrier, is relatively well understood. Survival probability of compound nucleus can be calculated in a statistical model. The second process, forming a compound nucleus (fusion probability, $P_{\text {fus }}$ ), is not well understood, and to understand this process is the subject of this research program.

We have studied reactions using actinide target nucleus, ${ }^{238} \mathrm{U}$. Actinide nuclei have a prolate deformation, which should influence the fusion probability. At the collision on the polar sides the Coulomb

\footnotetext{
ae-mail: nishio.katsuhisa@jaea.go.jp
} 
barrier is low, and the reaction starts from a distant configuration. Collision on the equatorial side has higher Coulomb barrier, and the reaction starts form the compact touching distance. We studied the orientation effects on fusion and/or quasifission by measuring the fission fragment mass distributions. With the analysis using a fluctuation dissipation model, fusion probability is determined. Validity of the proposed method to determine the fusion probability was confirmed by measuring the evaporation residue cross sections for seaborgium and hassium isotopes produced in the ${ }^{30} \mathrm{Si}+{ }^{238} \mathrm{U}$ and ${ }^{34} \mathrm{~S}+$ ${ }^{238} \mathrm{U}$ reactions, respectively.

\section{Experimental methods}

Fission fragment mass distributions in the reactions of ${ }^{30} \mathrm{Si},{ }^{31} \mathrm{P},{ }^{34,36} \mathrm{~S},{ }^{40} \mathrm{Ar},{ }^{48} \mathrm{Ca}+{ }^{238} \mathrm{U}$ were measured using beams supplied by the tandem accelerator of the Japan Atomic Energy Agency (JAEA) at Tokai. The experimental set-up and the analysis method were described in [4]. The beam intensities were typically from 0.1 to $1.0 p$-nA. The ${ }^{238} \mathrm{U}$ target with about $100 \mu \mathrm{g} / \mathrm{cm}^{2}$ thick was prepared by electrodeposition of $\mathrm{UO}_{2}$ on a nickel backing. Both fission fragments (FFs) were detected in coincidence by position-sensitive multiwire proportional counters (MWPCs) having an active area of $200 \mathrm{~mm} \times 120 \mathrm{~mm}$. The MWPCs covered the emission angle of $\pm 25.0^{\circ}$ around the detector center. Fission events occurring after complete transfer of the projectile momentum to the composite system (full momentum transfer (FMT) fission) were separated from those fission events following nuclear transfer by recording the folding angle formed by two fission fragments.

\section{Experimental results and discussions}

The measured fission fragment mass distributions in the reactions of ${ }^{30} \mathrm{Si},{ }^{31} \mathrm{P},{ }^{36} \mathrm{~S},{ }^{40} \mathrm{Ar},{ }^{48} \mathrm{Ca}+$ ${ }^{238} \mathrm{U}$ are shown in Fig.1 [5-7]. In each reaction, data at four incident energy points are shown. In the ${ }^{30} \mathrm{Si},{ }^{31} \mathrm{P}$ and ${ }^{36} \mathrm{~S}$-induced reactions, we observed a mass-symmetric distribution at the highest incident energy. The mass-asymmetric fission channel appears at the low energies. The variation of the measured distributions with incident energy is interpreted by the effects of nuclear orientation on fusion and/or quasifission. At the lowest incident energies, the reaction is limited to the collision on the polar sides of the nucleus ${ }^{238} \mathrm{U}$. This configuration leads to quasifission with higher probability than the reaction starting from equatorial collisions. Quasifission probability generally increases with the mass and/or charge of projectile nucleus.

In order to make a quantitative analysis of the mass distribution and to determine the fusion probability $P_{\text {fus }}$, we performed a model calculation combining the coupled channels method and a dynamical description of the reaction based on the three-dimensional Langevin equation [8]. The dynamical calculation based on the Monte Carlo method was used for describing the reaction paths in the potential energy landscape. The deformation of the reaction partners and their statistical orientation in the reaction plane was considered. Fusion-fission is defined as the fission after forming the compound nucleus. Quasifission is the binary decay without reaching the compound nucleus shape.

The measured mass mass distributions are compared with the model calculation in the reactions of ${ }^{30} \mathrm{Si}+{ }^{238} \mathrm{U}$ and ${ }^{34} \mathrm{~S}+{ }^{238} \mathrm{U}$ in Fig.2. Good agreement is found at entire energy range for both reactions. Especially, appearance of the asymmetric fission at the low incident energies is well demonstrated. Fusion-fission events were chosen in the trajectory analysis, and the corresponding mass distributions are also shown in Fig.2. Fusion-fission spectrum shows the mass-symmetric shape. Fusion probability $P_{\text {fus }}$ is obtained from the fraction of fusion-fission events. In the reaction of ${ }^{30} \mathrm{Si}+{ }^{238} \mathrm{U}, P_{\text {fus }}$ decreases from $46 \%$ to $29 \%$ toward the low incident energy. The probability changes from $15 \%$ to $3.6 \%$ in the ${ }^{34} \mathrm{~S}+{ }^{238} \mathrm{U}$ reaction. 


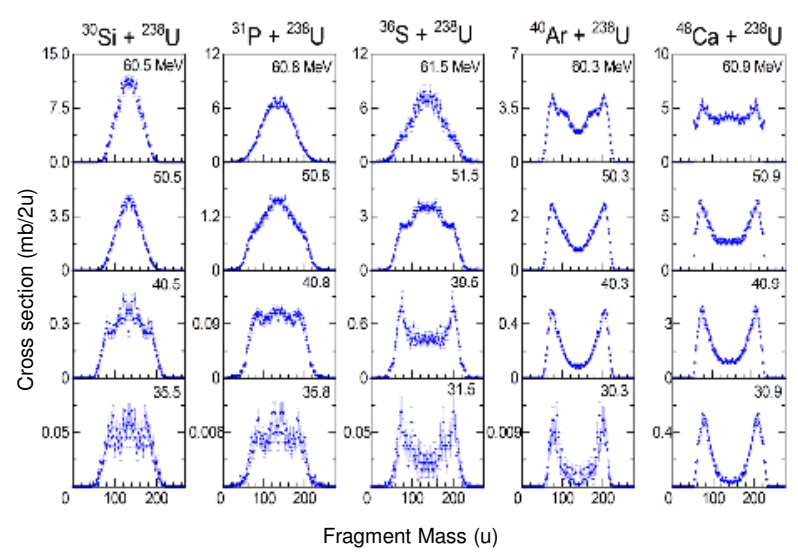

Figure 1. Fission fragment mass distributions of full momentum transfer fission. The numerical value in each section of the figure show the excitation energy of the compound nucleus.

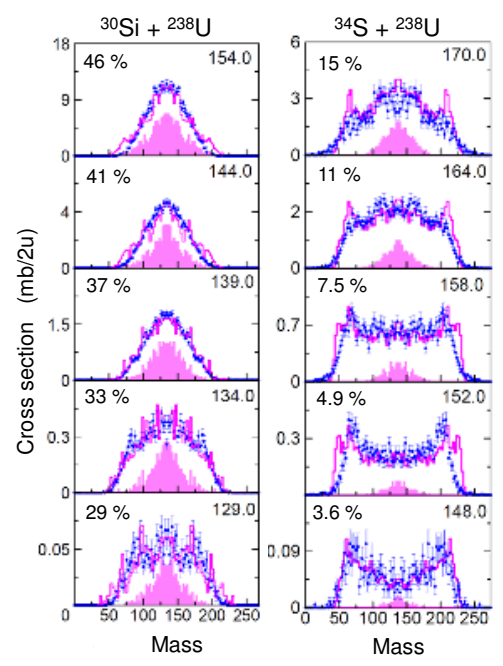

Figure 2. Fission fragment mass distributions for ${ }^{30} \mathrm{Si}+{ }^{238} \mathrm{U}$ and ${ }^{34} \mathrm{~S}+{ }^{238} \mathrm{U}$. Numerical value marked at the right-top position is the center-of-mass energy. The histogram includes all fission fragments in the calculation. Fusion-fission spectra in the calculation are shown by filled histograms. Fusion probabilities are shown in $\%$ for each energy points.

Validity of the obtained fusion probability was confirmed by measuring the evaporation residue cross sections. Experiment to produce seaborgium and hassium isotopes produced in ${ }^{30} \mathrm{Si}+{ }^{238} \mathrm{U}$ and ${ }^{34} \mathrm{~S}+{ }^{238} \mathrm{U}$ were carried out at GSI in Darmstadt [6,9]. The velocity filter SHIP [2] was used to separate evaporation residues from the primary beams and other reaction products. Figure 3 summarizes the capture cross sections, fusion cross sections and evaporation residue cross sections for both reactions. The cross sections for ${ }^{263,264} \mathrm{Sg}$ and ${ }^{267,268} \mathrm{Hs}$ were compared with a statistical model calculation, 


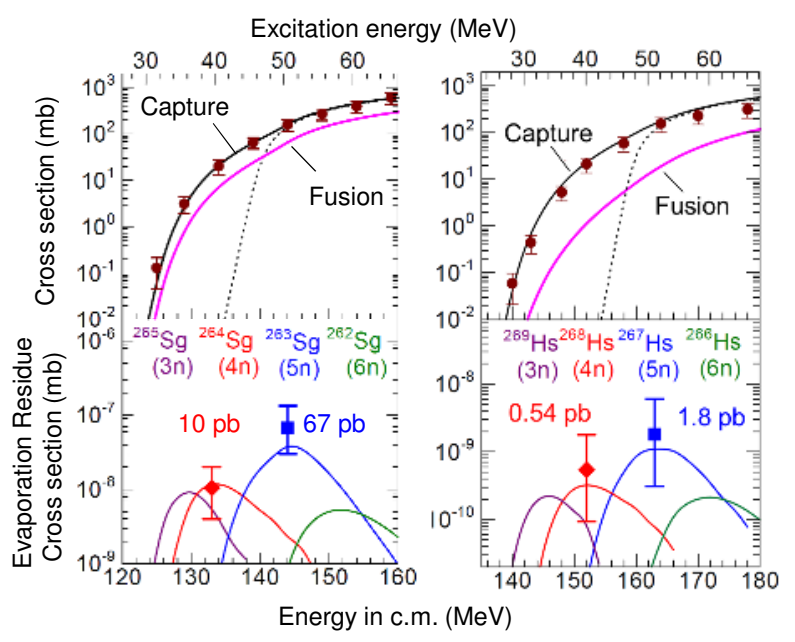

Figure 3. (Upper panel) Measured fission cross sections of the reaction ${ }^{30} \mathrm{Si}+{ }^{238} \mathrm{U}$ (left) and ${ }^{34} \mathrm{~S}+{ }^{238} \mathrm{U}$ (right) as a function of the center-of-mass energy and the excitation energy $\left(E^{*}\right)$ of the compound nucleus. Solid curve (black) show the capture cross-section calculated by the coupled-channels model. Dotted curve is the calculation using one-dimensional barrier penetration model. Solid curve (magenta) is the fusion cross section determined by multiplying the fusion probability to the capture cross section. (Lower panel) Measured cross sections for ${ }^{263,264} \mathrm{Sg}$ and ${ }^{267,268} \mathrm{Hs}$ are compared to the statistical model calculations.

where the obtained fusion probabilities were used as input for the HIVAP code [10]. The measured cross sections agree with the calculation.

\section{Summary}

We proposed to determine the fusion probability in the measurement of fission fragment mass distributions and analysis using the fluctuation dissipation model. Taking advantage of the lowering of Coulomb barrier in the polar collisions, there are probability to produce evaporation residues at sub-barrier energies by evaporating lower number neutrons [5-7].

\section{References}

[1] Yu. Ts. Oganessian, J. Phys. G 34, R165 (2007).

[2] S. Hofmann and G.Munzenberg, Rev. Mod. Phys. 72, 733 (2000).

[3] K. Morita et al., J. Phys. Soc. Jpn. 81, 103201 (2012).

[4] K. Nishio et al., Phys. Rev. C 77, 064607 (2008).

[5] K. Nishio et al., Phys. Rev. C 82, 044604 (2010).

[6] K. Nishio et al., Phys. Rev. C 82, 024611 (2010).

[7] K. Nishio et al., Phys. Rev. C 86, 034608 (2012).

[8] Y. Aritomo et al., Phys. Rev. C 85, 044614 (2012).

[9] K. Nishio et al., Eur. Phys. J. A 29, 281 (2006).

[10] W. Reisdorf and M. Schädel, Z. Phys. A 343, 47 (1992). 